Audiology

Neurotology
Audiol Neurotol 2018;23:356-363

DOI: $10.1159 / 000495132$
Received: March 26, 2018

Accepted after revision: October 31, 2018

Published online: February 8, 2019

\title{
A Prospective Study of the Effect of Cochlear Implantation on Tinnitus
}

\author{
Francka J.J. Kloostra Julia Verbist Rutger Hofman Rolien H. Free \\ Rosemarie Arnold Pim van Dijk
}

Department of Otorhinolaryngology and Head and Neck Surgery, University Medical Center Groningen, Groningen, The Netherlands

\section{Keywords}

Tinnitus · Cochlear implantation $\cdot$ Handicap

\begin{abstract}
Previous studies have shown diverse and sometimes even contrary results concerning the effect of cochlear implantation on tinnitus and the factors that can influence this effect. The aim of this prospective questionnaire study was to determine the effects of cochlear implantation on tinnitus and explore which factors can influence the effect of cochlear implantation on tinnitus. Forty-four of the patients implanted in our hospital returned 2 questionnaire packages, i.e., one before the cochlear implantation and one 6 months after implantation. Before implantation, $66 \%$ of the patients experienced tinnitus. This study shows that cochlear implantation could help to reduce tinnitus and the tinnitus handicap in at least $28 \%$ of the patients with preoperative tinnitus. In $72 \%$ of the patients the tinnitus remained after implantation. None of the patients developed tinnitus after implantation. A shorter duration of tinnitus prior to implantation, a more fluctuating type of tinnitus, a higher tinnitus handicap prior to implantation, and a round-window surgical approach might have a positive influence on the effect of cochlear implantation on tinnitus, but further research is necessary to confirm these findings. @2019 The Author(s)

Published by S. Karger AG, Basel
\end{abstract}

\section{KARGER}

E-Mail karger@karger.com www.karger.com/aud

(C) 2019 The Author(s)

Published by S. Karger AG, Basel

Karger

Open access

This article is licensed under the Creative Commons AttributionNonCommercial-NoDerivatives 4.0 International License (CC BY NC-ND) (http://www.karger.com/Services/OpenAccessLicense). Usage and distribution for commercial purposes as well as any distribution of modified material requires written permission.

\section{Introduction}

Tinnitus is a very common problem that has existed for centuries; the oldest known written report dates back to the ancient Egyptians and Mesopotamians [Stephens, 1984]. The prevalence of tinnitus in the general population ranges from 5 to $43 \%$, with $12-30 \%$ of these patients suffering from tinnitus that lasts for more than $5 \mathrm{~min}$ at a time [McCormack et al., 2016]. Of the patients who visit self-help groups because of their tinnitus, $30-42 \%$ are willing to use devices if this will significantly reduce their tinnitus, $52-62 \%$ are willing to use medication for tinnitus reduction, and $13-38 \%$ are even willing to undergo invasive procedures to obtain a significant tinnitus reduction [Tyler, 2012]. Although tinnitus is a phenomenon that has existed for centuries, its exact cause is still not known. There are several hypotheses about the cause of tinnitus. Many hypotheses have in common that central neural mechanisms play a key role in its development. Most of these hypotheses state that tinnitus is caused by maladaptive plastic changes in the central auditory pathways as a reaction to peripheral hearing loss [Cacace et al., 2016].

Within the population of cochlear-implant candidates, the prevalence of tinnitus is between 66 and $86 \%$ [Hazell et al., 1995; Quaranta et al., 2004; Baguley and 
Atlas, 2007; Andersson et al., 2009; Kompis et al., 2012]. This high prevalence is consistent with the fact that people with hearing impairment are more likely to suffer from tinnitus [Han et al., 2009]. Cochlear implantation is associated with a postoperative reduction of tinnitus loudness and awareness [Ruckenstein et al., 2001; Di Nardo et al., 2007]. Previous studies have shown a positive effect of cochlear implantation on both tinnitus prevalence and the handicap it causes in up to $93 \%$ of patients [Ruckenstein et al., 2001; Pan et al., 2009; Kloostra et al., 2015]. Unfortunately, cochlear implantation can also have a negative effect on tinnitus. In some studies, participants have experienced deterioration of their tinnitus or have even developed tinnitus [Kompis et al., 2012; Kloostra et al., 2015; van Zon et al., 2016]. According to previous studies, the number of participants that experience deterioration of tinnitus after cochlear implantation is between 4 and 26\% [Kompis et al., 2012].

As cochlear implantation can be of value in reducing both the prevalence of tinnitus and the tinnitus handicap in patients who suffer from severe hearing problems, the aim of this prospective questionnaire study was to determine the effects of cochlear implantation on tinnitus and explore which factors can influence the effect of cochlear implantation on tinnitus. Previous studies have shown very diverse results concerning the effect of cochlear implantation on tinnitus and the factors that can influence this effect [Ruckenstein et al., 2001; Pan et al., 2009; Kompis et al., 2012; Kloostra et al., 2015; van Zon et al., 2016].

For our study, we used several validated questionnaires that enabled us to have a broad view of the effect that cochlear implantation might have on patients who suffer from tinnitus. We also used tinnitus handicap questionnaires to determine how the presence of tinnitus influences daily life in cochlear implant patients. We used a hearing handicap questionnaire to establish whether cochlear implantation, besides improving phoneme scores, also improves hearing abilities in daily life. Last, we included an anxiety and depression questionnaire to assess the influence of tinnitus and hearing loss on these feelings.

\section{Materials and Methods}

\section{Procedure and Inclusion}

A total of 63 successive CI candidates from the Cochlear Implant North Netherlands (CINN) patient database were asked to participate in this questionnaire study on tinnitus. All of the patients were implanted in the University Medical Center Groningen
(UMCG) between 2010 and 2013. All of them were 18 years or older at the time of this study and gave their written, informed consent. As concluded by the Medical Ethics Committee of the UMCG, this study it not subject to Dutch law on Medical-Scientific Research on Humans (Wet Medisch-wetenschappelijk Onderzoek met Mensen, WMO). This study was conducted in accordance with the Declaration of Helsinki and applicable Dutch laws.

\section{Surgical Techniques}

Two surgical approaches, i.e., the round-window insertion technique and the cochleostomy technique, were used for cochlear implantation. In the first technique, electrode insertion is done through the round-window aperture. In the second technique, electrode insertion is also done through the round-window aperture, but the round window is enlarged using a small drill. It is thought that this round-window insertion technique causes less damage to the cochlea. Most commonly, surgeons use the cochleostomy technique instead of the round-window insertion technique due to the sort of electrode that was implanted. The CI422 electrode can be placed using the round-window insertion technique and the CI24RE electrode and the Advanced Bionics electrode can only be placed using the cochleostomy technique. In fewer cases, the reason for using the cochleostomy technique is that the position of the facial nerve makes it impossible to reach the round window.

\section{Measures}

In this prospective study, we sent the participating patients 2 questionnaire booklets. The first booklet was sent when the participant agreed to undergo cochlear implantation. It was thus completed weeks to a couple of months prior to the implantation. This booklet consisted of questions about the preoperative situation. The second booklet was completed 6 months after the patient was implanted and consisted of questions about the postoperative situation. Each booklet contained several questionnaires about tinnitus characteristics, depression/anxiety, and hearing status. Also, the UMCG anamnestic tinnitus questionnaire was included, which contained several questions about the characteristics of the tinnitus and the influence of the CI on tinnitus perception. Further information about audiological tests, the date of implantation, the surgery method, and the processor type was collected from the clinical files of the patients.

\section{Questionnaires}

Abbreviated Profile of Hearing Aid Benefit

We used the translated and validated Dutch version of the $A b$ breviated Profile of Hearing Aid Benefit (APHAB). The APHAB questionnaire assesses hearing ability and discomfort [Cox and Alexander, 1995]. It contains 24 questions that are divided into the following 4 subscales: ease of communication (EC), background noise $(\mathrm{BN})$, reverberation (RV), and aversiveness (AV). The EC scale describes the ability to understand people in various situations. The BN scale describes how background noise affects the ability to understand conversations. The RV scale describes the influence of reverberation/echo in rooms on hearing ability. The AV scale describes the discomfort caused by unpleasant sounds. All of these subscales contain 6 items and the patient is asked to rate all of the questions according to how often situations occur. The 7 different gradations are as follows: always (99\% of the time), almost always (87\%), generally (75\%), half the time (50\%), occasionally (25\%), seldom (12\%), and never (1\%). 
Hospital Anxiety and Depression Scale

We used the translated and validated Dutch version of the Hospital Anxiety and Depression Scale (HADS) [Spinhoven et al., 1997]. The HADS questionnaire was developed to improve the early detection of patients with any form of an anxiety or depression disorder [Zigmond and Snaith, 1983]. The questionnaire contains 14 items, i.e., 7 about anxiety and 7 about depression. Patients answer the questions on a scale of $0-3$. Subscale scores can be calculated for anxiety and depression. Scores below 7 indicate neither an anxiety nor a depression problem, scores between 8 and 10 suggest, a potential anxiety or depression disorder, and scores beyond 11 indicate definite cases.

Tinnitus Handicap Inventory

We used the translated and validated Dutch version of the Tinnitus Handicap Inventory (THI). The THI questionnaire consists of 25 questions about the influence of tinnitus on activities of daily life [Newman et al., 1996]. The questionnaire contains 11 questions about the functional effects on tinnitus, 9 questions about the emotional responses to tinnitus, and 5 questions about catastrophic responses to tinnitus. Patients have to indicate if such situations occur. Every "yes" counts for 4 points, every "sometimes" for 2 points, and every "never" for 0 points. The scores are summed to produce a total score between 0 and 100 . Scores below 16 have been suggested to imply a slight handicap, scores from 18 to 38 indicate mild tinnitus, scores from 38 to 56 correspond to severe tinnitus, and scores higher than 78 mean that the patient suffers from catastrophic tinnitus [McCombe, 2001].

Tinnitus Handicap Questionnaire

The Tinnitus Handicap Questionnaire (THQ) is used to measure the perception of psychological and physical well-being in tinnitus patients. In this study, we used a Dutch translation of the questionnaire [Vanneste et al., 2011]. It contains 27 questions divided into the following 3 main categories: social, emotional, and behavioral tinnitus effects; tinnitus and hearing; and outlook on tinnitus. Patients answer whether or not they agreed with the statements on a scale of $0-100$. Total scores are calculated per category, after which the mean THQ score is calculated [Kuk et al., 1990].

UMCG Anamnestic Tinnitus Questionnaire

This is a nonvalidated questionnaire that concerns tinnitus characteristics such as pitch, loudness, and lateralization [Kloostra et al., 2015].

\section{Statistical Analysis}

Pre- and postoperative questionnaire scores were analyzed and compared to identify significant differences in tinnitus and related patient parameters before and after cochlear implantation. We used the Kolmogorov-Smirnov test and histograms to assess whether variables met the criteria for a normal distribution. If a variable was normally distributed, we used a paired $t$ test to identify significant differences between pre- and postoperative scores within a group of patients. If the data were not normally distributed, we used the Wilcoxon signed-rank test to determine whether there was a significant difference. To compare differences between the separated groups in a not normally distributed population, the Mann-Whitney U test was used. For all statistical analyses we used SPSS version 23.

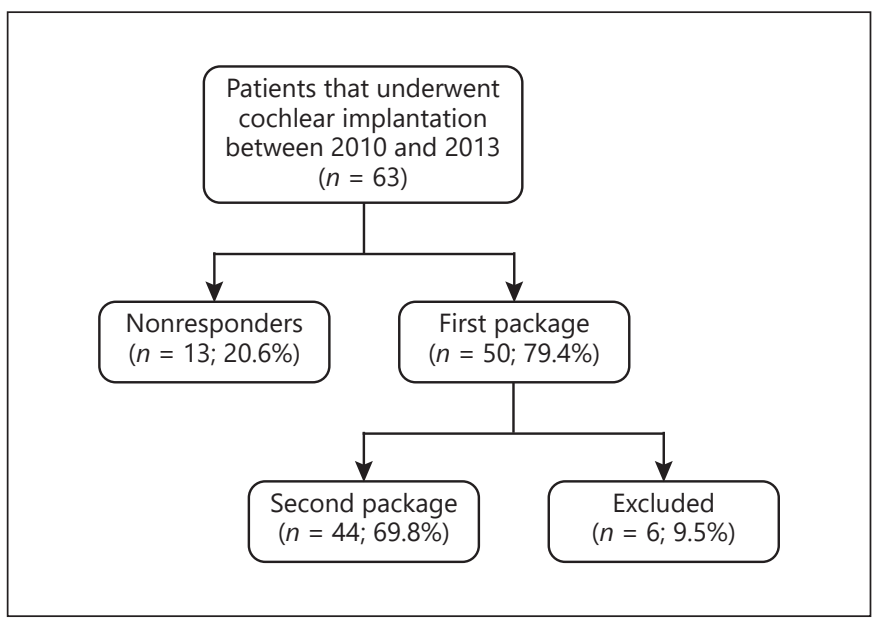

Fig. 1. Inclusion flowchart. Reasons for exclusion were: nonresponders, patient who did not undergo cochlear implantation, and patients who died.

\section{Results}

\section{Participants}

Of the 63 patients we contacted, 50 subjects $(79.4 \%, 26$ males and 24 females) returned the first package of questionnaires. Forty-seven of these participants received a cochlear implant in our hospital. Three patients were not implanted; 1 died while on the waiting list, 1 decided not to be implanted, and 1 was implanted in another hospital. Forty-four of the implanted patients also returned the postoperative package of questionnaires (Fig. 1).

Before cochlear implantation, 33 out of 50 participants (66.0\%) reported tinnitus. Postoperatively, 21 out of 44 participants $(47.7 \%)$ reported tinnitus. Twenty-one $(47.7 \%)$ of the 44 participants who returned both the preoperative and the postoperative questionnaire booklets suffered from both pre- and postoperative tinnitus, 8 participants $(18.2 \%)$ of these only experienced tinnitus before the implantation, and 15 participants (34.1\%) never experienced tinnitus. None of the participants developed tinnitus after implantation.

\section{Background Variables}

Figure 2 shows the results from the 44 participants who returned both the preoperative and the postoperative questionnaire booklets.

Focusing on the group of patients with preoperative tinnitus, $72.4 \%$ suffered from both post- and preoperative tinnitus and $27.6 \%$ suffered only from preoperative tinnitus. The mean number of years participants experi- 


\begin{tabular}{|c|c|c|c|c|c|c|c|c|}
\hline & \multicolumn{2}{|c|}{ Patients without tinnitus } & \multicolumn{2}{|c|}{ All patients with preoperative tinnitus } & \multicolumn{2}{|c|}{ Only preoperative tinnitus } & \multicolumn{2}{|c|}{ Both pre- and postoperative tinnitus } \\
\hline $\begin{array}{l}\text { Number of patients } \\
\text { Percentage }\end{array}$ & \multicolumn{2}{|c|}{15} & \multicolumn{2}{|c|}{$\begin{array}{r}29 \\
100 \\
\end{array}$} & \multicolumn{2}{|c|}{$\begin{array}{c}8 \\
27.6 \\
\end{array}$} & \multicolumn{2}{|c|}{$\begin{array}{l}21 \\
72.4\end{array}$} \\
\hline Gender (male/female), $\mathrm{n}$ & \multirow{5}{*}{\multicolumn{2}{|c|}{$\begin{array}{c}8 / 7(n=15) \\
62.8(S D 14.8, n=15) \\
10 / 5(n=15) \\
14 / 1(n=15) \\
7 / 8(n=15)\end{array}$}} & \multirow{6}{*}{\multicolumn{2}{|c|}{$\begin{array}{c}14 / 15(n=29) \\
64.2(S D 14.9, n=29) \\
14 / 11(n=29) \\
26 / 3(n=29) \\
15 / 13(n=28) \\
24.7(S D 22.3, n=18)\end{array}$}} & \multirow{6}{*}{\multicolumn{2}{|c|}{$\begin{array}{c}2 / 6(n=8) \\
60.0(5 \mathrm{D} 20.1, \mathrm{n}=8) \\
5 / 3(\mathrm{n}=8) \\
7 / 1(\mathrm{n}=8) \\
2 / \mathrm{n}(\mathrm{n}) 8) \\
5.2(\mathrm{SD} 5.2, \mathrm{n}=5)\end{array}$}} & \multirow{6}{*}{\multicolumn{2}{|c|}{$\begin{array}{c}12 / 9(\mathrm{n}=21) \\
66.0(\mathrm{SD} 12.6, \mathrm{n}=21) \\
9 / 12(\mathrm{n}=21) \\
19 / 2(\mathrm{n}=21) \\
13 / 7(\mathrm{n}=20) \\
32.2(\mathrm{SD} 21.9, \mathrm{n}=13)\end{array}$}} \\
\hline Age (at implantation) & & & & & & & & \\
\hline $\mathrm{Cl}$ ear (right/left), $n$ & & & & & & & & \\
\hline Brand: (Cochlear/Advanced Bionics), $\mathrm{n}$ & & & & & & & & \\
\hline Operation method: (cochleostomy/round window), $n$ & & & & & & & & \\
\hline \multirow{3}{*}{$\begin{array}{l}\text { Years of preoperative tinnitus } \\
\text { Percent of the time tinnitus preop/postop } \\
\text { Number of days tinnitus per month preop/postop }\end{array}$} & & & & & & & & \\
\hline & . & - & $76.1($ SD $30.1, n=28)$ & - & $65.9(\mathrm{SD} 31.2, \mathrm{n}=8)$ & - & $80.3($ SD 26.7, $n=20)$ & $67.2(\mathrm{SD} 32.1, \mathrm{n}=18)^{\star}$ \\
\hline & & - & $28.0($ SD $6.6, n=28)$ & & $26.6($ SD $9.5, n=8)$ & - & 28.6 (SD 5.2, $\mathrm{n}=20)$ & $27.9(\mathrm{SD} 6.3, \mathrm{n}=16)$ \\
\hline THI preop/postop & 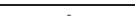 & - & $23.6(\mathrm{SD} 21.2, \mathrm{n}=29)$ & - & $31.8(\mathrm{SD} 26.2, \mathrm{n}=8)$ & - & $20.5($ SD $18.9, n=21)$ & $13.3(\mathrm{SD} 13.6, \mathrm{n}=18)$ \\
\hline THQ preop/postop & & . & $30.7($ SD 11.0, $n=23)$ & - & $31.8($ SD $13.0, \mathrm{n}=5)$ & - & $30.3($ SD $10.8, n=18)$ & $20.9($ SD $18.7, \mathrm{n}=17)$ \\
\hline $\begin{array}{l}\text { Side of tinnitus preoperative number left/right//both } \\
\text { Side of tinnitus postoperative } \mathrm{Cl} \text { on number left/right//both } \\
\text { Side of tinnitus postoperative } \mathrm{Cl} \text { off number left/right//both }\end{array}$ & & & \multicolumn{2}{|l|}{$2 / 1 / 31$} & & & & $\begin{array}{l}1 / 3 / 13 \\
2 / 3 / 15\end{array}$ \\
\hline Sound of tinnitus preoperative number diverse/whistle/tonal & & & \multirow{2}{*}{\multicolumn{2}{|c|}{$26 / 4 / 1$}} & & & & \\
\hline $\begin{array}{l}\text { Sound of tinnitus postoperative } \mathrm{Cl} \text { on number diverse/whistle/tonal } \\
\text { Sound of tinnitus postoperative } \mathrm{Cl} \text { off number diverse/whistle/tonal }\end{array}$ & & & & & & & & $\begin{array}{l}14 / 2 / 0 \\
17 / 3 / 0\end{array}$ \\
\hline $\begin{array}{l}\text { Sound of tinnitus postoperative Cl off number diverse/whistle/tonall } \\
\text { Tinnitus influence on sleep preoperative number yes/sometimes/no }\end{array}$ & & & \multirow{2}{*}{\multicolumn{2}{|c|}{$5 / 15 / 13$}} & & & & \\
\hline Tinnitus influence on sleep postoperative number yes/sometimes/no & & & & & & \multirow{4}{*}{\multicolumn{2}{|c|}{ 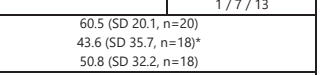 }} \\
\hline \begin{tabular}{|c|} 
Loudness preoperative \\
\end{tabular} & \multirow{3}{*}{\multicolumn{2}{|c|}{-}} & \multirow{3}{*}{\multicolumn{2}{|c|}{$\frac{1}{46.6(S D 22.8, n=26)}$}} & \multirow{3}{*}{\multicolumn{2}{|c|}{$43.1($ SD 28.2, $n=8)$}} & & \\
\hline Loudness postoperative, $\mathrm{Cl}$ on (compared to preoperative situation) & & & & & & & & \\
\hline Loudness postoperative, $\mathrm{Cl}$ off (compared to preoperative situation) & & & & & & & & \\
\hline Pitch preoperative & \multirow{3}{*}{\multicolumn{2}{|c|}{-}} & \multirow{3}{*}{\multicolumn{2}{|c|}{$55.5($ SD 23.5, n=28) }} & \multirow{3}{*}{\multicolumn{2}{|c|}{$47.9($ SD 20.6, n=7) }} & \multirow{3}{*}{\multicolumn{2}{|c|}{$\begin{array}{l}46.2(S D 24.0, n=19) \\
45.9(S D \text { 34.0, } n=16) \\
55.0(S D \text { S0.4, } \mathrm{n}=16)\end{array}$}} \\
\hline Pitch postoperative, $\mathrm{Cl}$ on (compared to preoperative situation) & & & & & & & & \\
\hline Pitch postoperative, Cl off (compared to preoperative situation) & & & & & & & & \\
\hline Phonemescore preop/postop & 36.1 (SD 15.6, n=14) & $67.3(\mathrm{SD} 23.7, \mathrm{n}=15)^{\star \star}$ & 39.6 (SD 17.4, n=27) & $72.4(\text { SD 17.1, } n=27)^{\star \star}$ & 37.5 (SD 16.7, n=8) & $66.9(\text { SD } 20.0, n=8)^{\star * *}$ & 40.5 (SD 18.1, n=19) & 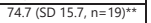 \\
\hline APHAB preop/postop & \multirow{2}{*}{\multicolumn{2}{|c|}{$\begin{array}{c}77.2(\mathrm{SD} 12, \mathrm{n}=15) \quad 44.9(\mathrm{SD} 18.7, \mathrm{n}=8)^{* *} \\
13 / 2(\mathrm{n}=15)\end{array}$}} & \multirow{2}{*}{\multicolumn{2}{|c|}{ 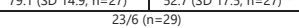 }} & \multirow{2}{*}{\multicolumn{2}{|c|}{ 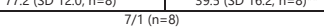 }} & \multirow{2}{*}{\multicolumn{2}{|c|}{$79.8(30) 16.2, n=19)(16 / 5(n=21)$}} \\
\hline Preoperative hearingaids (yes $/ n o), n$ & & & & & & & & \\
\hline Mean anxiety score, preop/postop & $3.1(\mathrm{SD} 3.8, \mathrm{n}=14)$ & $1.1(\mathrm{SD} 1.3, \mathrm{n}=14)^{*}$ & $5.3(\mathrm{SD} 3.6, \mathrm{n}=28)$ & $3.8(\mathrm{SD} 3.5, \mathrm{n}=28)^{\star}$ & $6.9(\mathrm{SD} 4.8, \mathrm{n}=8)$ & $3.9(\mathrm{SD} 3.9, \mathrm{n}=8)^{*}$ & $4.7($ SD $2.9, \mathrm{n}=20)$ & $3.7(\mathrm{SD} 3.4, \mathrm{n}=20)$ \\
\hline Mean depression score, preop/postop & $2.8(\mathrm{SD} 3.2, \mathrm{n}=14)$ & $2.9(\mathrm{SD} 3.5, \mathrm{n}=13)$ & $4.4(\mathrm{SD} 2.7, \mathrm{n}=28)$ & $3.2(\mathrm{SD} 3.6, \mathrm{n}=27)$ & $4.3(\mathrm{SD} 3.1, \mathrm{n}=8)$ & $4.1(\mathrm{SD} 4.7, \mathrm{n}=7)$ & $4.5(\mathrm{SD} 2.6, \mathrm{n}=20)$ & $2.9(\mathrm{SD} 3.1, \mathrm{n}=20)$ \\
\hline
\end{tabular}

Fig. 2. Patient characteristics and changes after CI implantation.

enced tinnitus before cochlear implantation was 24.7 (SD 22.3 , range 1-67). The group with only preoperative tinnitus experienced tinnitus during significantly fewer years prior to implantation (i.e., 5.2 years, SD 5.2, range 1-14]) compared to the group with both pre- and postoperative tinnitus (i.e., 32.2 years, SD 21.9, range 1-67) $(p<0.05)$. Participants with preoperative tinnitus experienced tinnitus $76.1 \%$ of the time (SD 30.1). This percentage was significantly higher in the group with both preand postoperative tinnitus (80.3\%, SD 26.7) compared to the group with only preoperative tinnitus $(65.9 \%, \mathrm{SD}$ $31.2)(p<0.05)$. Patients with preoperative tinnitus experienced their tinnitus on average 28 days a month (range 3-30).

Most patients experienced tinnitus in both ears or somewhere central in the head. Only a few patients experience tinnitus unilaterally. This was the case both for the preoperative and the postoperative situation (Fig. 2).

In both the preoperative and the postoperative situation, the tinnitus sounds perceived were diverse. Postoperatively no patient continued to perceive a tonal tinnitus sound.

Preoperatively almost half of the patients who answered this question experienced difficulty falling asleep because of their tinnitus. Postoperatively fewer patients experienced this difficulty because of their tinnitus (Fig. 2).

\section{Tinnitus Handicap}

In the participants with preoperative tinnitus, the mean preoperative THI score was 23 (SD 21.2), and the mean THQ score was 30.7 (SD 11.0). The group of participants with only preoperative tinnitus had higher preoperative scores on both the THI $(31.8 / 20.5, p=0.77)$ and the THQ $(31.8 / 30.3, p=0.19)$ compared to the THI and THQ scores of participants with both pre- and postoperative tinnitus. However, this difference was not significant.

Figure 3 shows the changes in tinnitus handicap in the group of participants with both pre- and postoperative tinnitus. The scatterplot shows the THI and THQ scores of all of the individuals in both the pre- and the postoperative situation. All dots above the line show deterioration in the tinnitus handicap, the dots below the line show improvement compared to the preoperative situation. Most of the dots (THI, 12 out of 17; THQ, 9 out of 14) are beneath the diagonal, which implies that the majority of the participants showed improvement in their THI and/ or THQ scores after cochlear implantation. Previous studies mention contrary results concerning the minimum change in the Tinnitus Handicap Inventory (THI) score that could be considered clinically relevant. Seven of the patients had a significant reduction of 7 points or more on the THI [Zeman et al., 2011] and only 1 patient had a significant reduction of 20 points or more on the THI [Newman et al., 1998].

According to Spearman's correlation test there was no significant correlation between postoperative scores on the THI and the THQ and postoperative phoneme scores, respectively (i.e., $-0.044, p=0.859$, for the THI and 0.209 , $p=0.404$, for the THQ). Also, there was no significant correlation between postoperative scores on the THI and 


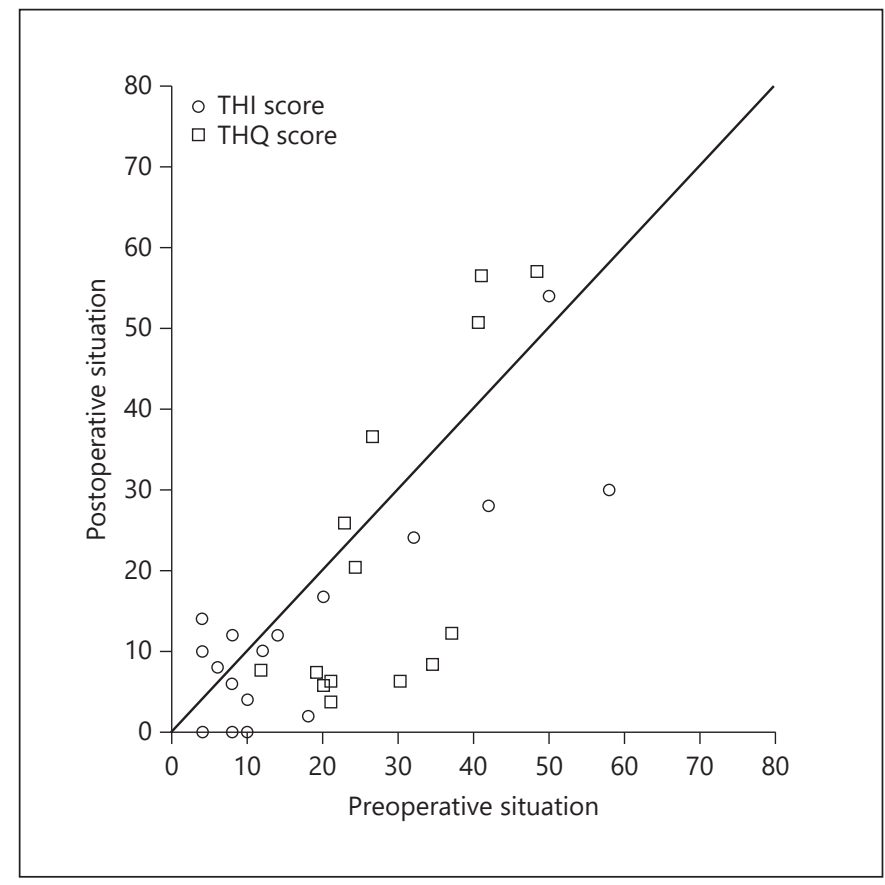

Fig. 3. Tinnitus handicap score. This scatter plot shows the preand postoperative THI and THQ scores of every participant who completed the corresponding questionnaire. Dots that are beneath the line show improvement in the tinnitus handicap, and dots above the line show deterioration of the tinnitus handicap. There are $16 \mathrm{THI}$ dots instead of 17 because 2 participants had the exact same pre- and postoperative scores (preoperatively, 6; postoperatively, 8).

postoperative scores on the APHAB (i.e., $0.336, p=0.148$ ). However, there was a significant correlation between postoperative scores on the THQ and postoperative scores on the APHAB (i.e., $0.529, p<0.05$ ).

\section{Loudness and Pitch}

In the anamnestic tinnitus questionnaire, tinnitus loudness and pitch were measured on a visual analogue scale with a range from 0 to 100 . The preoperative tinnitus loudness scored somewhat higher in the group that suffered from both pre- and postoperative tinnitus (i.e., 60.5 , SD 20.1) compared to the group with only preoperative tinnitus (i.e., 43.1, SD 28.2). However, this difference was not significant $(p=0.10)$. In the group of participants with both pre- and postoperative tinnitus the mean tinnitus loudness was significantly lower in the postoperative situation when the cochlear implant was turned on compared to the preoperative situation. This difference was not found when the cochlear implant was turned off.
When comparing the group with only preoperative tinnitus to the group with both pre- and postoperative tinnitus, no significant difference in preoperative pitch score was found $(p=0.91)$. The mean tinnitus pitch of participants with both pre- and postoperative tinnitus changed from 46.2 (SD 24.0) in the preoperative situation to 45.9 (SD 34.0) in the postoperative situation when the cochlear implant was turned on. When the cochlear implant was turned off, the postoperative pitch was somewhat higher (i.e., 55.0, SD 30.4). However, these differences were not significant.

\section{Surgical Details}

Twenty-four of the patients were implanted in the right ear and 20 in the left ear. Most of the participants received a device of the manufacturer Cochlear $(n=40)$, and the remaining participants received an Advanced Bionics implant $(n=4)$. As noted in Materials and Methods, 2 surgical implantation techniques were used, i.e., the cochleostomy technique (22 patients) and the round-window insertion technique (21 patients). In 1 patient, the technique used had not been recorded. Using the MannWhitney U test revealed no significant difference between surgical techniques between the groups with and without postoperative tinnitus $(p=0.06)$, but in the group where the tinnitus remained $65 \%$ of the patients were operated using the cochleostomy technique whereas in the group in which the tinnitus ceased only $25 \%$ of the patients were operated using this technique.

\section{Hearing Ability}

All groups of patients showed significant improvement in phoneme scores after cochlear implantation, which indicated that their hearing ability had improved. Also, the hearing handicap, as measured by the APHAB, was significantly lower in all of the groups.

\section{Anxiety and Depression}

\section{Anxiety}

Both in the group with $(5.3 / 3.8, p<0.05)$ and the group without $(3.1 / 1.1, p<0.05)$ preoperative tinnitus, the mean anxiety scores were significantly lower postoperatively compared to preoperatively. The postoperative anxiety score (i.e., 3.9; SD 3.9) was also significantly lower than the preoperative score (i.e., 6.9; SD 4.8) in the group of patients with only preoperative tinnitus. This difference was not significant in the group with both pre- and postoperative tinnitus; it was 4.7 (SD 2.9) preoperatively and 3.7 (SD 3.4) postoperatively. 
Clinical HADS scores $>8$ were present in 2 patients in the preoperative situation. Both of these patients suffered only from preoperative- and not postoperative tinnitus. One of these patients still had a score $>8$ in the postoperative situation. None of the other patients scored higher than 8 after cochlear implantation.

\section{Depression}

Both in the group with (4.4/3.2) and in the group without $(2.8 / 2.9)$ preoperative tinnitus there was no significant difference in the mean depression score preoperatively compared to postoperatively. Moreover, no significant differences were found in the group with both pre- and postoperative tinnitus or with only preoperative tinnitus. One patient scored $>8$ on the HADS preoperatively and had suffered from both pre- and postoperative tinnitus. There were 2 patients who scored $>8$ on the HADS after implantation. One of them only suffered from preoperative tinnitus and the other suffered from both pre- and postoperative tinnitus. Neither of these patients was the one person with a high preoperative depression score.

\section{Discussion}

In this prospective study, we investigated the influence of cochlear implantation on tinnitus in patients who received a cochlear implant because of severe hearing loss. Our study showed that in $27.6 \%$ of the patients with preoperative tinnitus the tinnitus disappeared after cochlear implantation. In the group of patients in whom the tinnitus remained after cochlear implantation, most of the patients showed lower tinnitus handicap scores and lower tinnitus loudness scores after the operation. The patients in the group in which the tinnitus disappeared had fewer years of tinnitus prior to implantation, experienced tinnitus for less time during the day prior to implantation, experienced more of a tinnitus handicap prior to implantation, and were more often operated on using the round-window approach compared to the patients whose the tinnitus remained.

Our result that tinnitus cessation occurred in $27.6 \%$ $(n=8)$ of the patients with preoperative tinnitus is in line with the numbers described in previous studies, which report suppression of tinnitus after cochlear implantation in 15-95\% of the patients [Rubinstein et al., 2003; Quaranta et al., 2008; Van de Heyning et al., 2008; Akdogan et al., 2009; Pan et al., 2009; Bovo et al., 2011; Kompis et al., 2012]. Our percentage is somewhat low in this range, probably because our number only describes the patients

Effects of Cochlear Implantation on

Tinnitus with tinnitus cessation and does not include the patients who experienced improvement of their tinnitus. Figure 3 shows that also about two thirds of the 21 patients with persistent tinnitus after cochlear implantation had lower tinnitus handicap scores after cochlear implantation, so we estimate that the percentage of patients with at least partial tinnitus suppression after cochlear implantation is close to $75 \%(n=8+14=21 / 29)$.

In the group of participants in whom tinnitus did not disappear in the postoperative situation, the mean time they experienced tinnitus prior to implantation was 32.2 years (SD 21.9, range 1-67). In the participants without postoperative tinnitus this was only 5.2 years (SD 5.2, range 1-14). The duration of suffering from tinnitus prior to implantation may influence the chance of the tinnitus disappearing with cochlear implantation. This finding can be explained by the theory that tinnitus results from an increase in spontaneous neural activity and plastic reorganization of the central nervous system. It is possible that these neural changes are harder to undo when they have existed for a longer time before treatment is started, because of less plasticity. There are no previous studies reporting that the duration of tinnitus prior to implantation influences the effect of cochlear implantation on tinnitus. However, there are previous studies reporting that the number of years of hearing loss prior to implantation can have this influence [Olze et al., 2011a, b].

Our study also shows that patients with tinnitus cessation after cochlear implantation experience tinnitus for less time per day compared to patients in whom the tinnitus remained after implantation. This could mean that patients with tinnitus cessation after cochlear implantation suffer more from fluctuating tinnitus, while patients in whom the tinnitus remains suffer more from a continuous tinnitus. Possibly, a more fluctuating tinnitus is more easily treated than a more continuous tinnitus because the changes in the central nervous system could be less prominent in patients with a fluctuating tinnitus. However, no other studies have confirmed these results.

The participants who experienced tinnitus cessation also experienced a higher preoperative tinnitus handicap (both THI and THQ) than the participants who still had tinnitus in the postoperative situation. This suggests that the more inconvenience is experienced from tinnitus before cochlear implantation, the more likely it is that the tinnitus will cease after implantation. This finding has also been described in previous studies [Kim et al., 2013; Ramakers et al., 2015].

The surgical approach is another possible predictive factor for the effect of cochlear implantation on tinnitus. 
in the group without postoperative tinnitus, most of the participants $(75.0 \%)$ were operated on using round-window insertion. In the group that still experienced tinnitus postoperatively, this was only $35.0 \%$. This finding suggests that using round-window insertion increases the chance that the tinnitus will disappear after cochlear implantation, compared to use of a separate cochleostomy. An explanation for this finding is that surgery using round-window insertion with a slimmer electrode may cause less damage to the cochlea and hair cells. Thus, this technique offers a higher chance of preserving the residual hearing of the patients and diminishes the chance of further reduction of the afferent input to the brain, and thereby maintaining or worsening the tinnitus.

\section{Conclusion}

Tinnitus is a very common problem of cochlear implant candidates and cochlear implant users. This study shows that cochlear implantation can help to reduce the tinnitus and the tinnitus handicap. A shorter duration of tinnitus prior to implantation, a tonal tinnitus, a higher tinnitus handicap prior to implantation, and a roundwindow insertion operation technique correlate with a positive influence of cochlear implantation on tinnitus. Further research is necessary in a larger and a more uniform study population with regard to hearing abilities, age, and type of tinnitus (unilateral/tonal).

\section{Statement of Ethics}

This study was approved by the Medical Ethics Committee of the UMCG.

\section{Disclosure Statement}

The authors report no conflict of interests.

\section{References}

Akdogan O, Ozcan I, Ozbek C, Dere H. Tinnitus after cochlear implantation. Auris Nasus Larynx. 2009 Apr;36(2):210-2.

Andersson G, Freijd A, Baguley DM, Idrizbegovic E. Tinnitus distress, anxiety, depression, and hearing problems among cochlear implant patients with tinnitus. J Am Acad Audiol. 2009 May;20(5):315-9.

Baguley DM, Atlas MD. Cochlear implants and tinnitus. Prog Brain Res. 2007;166:347-55.

Bovo R, Ciorba A, Martini A. Tinnitus and cochlear implants. Auris Nasus Larynx. 2011 Feb;38(1):14-20.

Cacace AT, de Kleine E, Holt AG, van Dijk P. Scientific foundations of audiology, perspectives from physics, biology, modeling and medicine. San Diego: Plural Publishing; 2016.

Cox RM, Alexander GC. The abbreviated profile of hearing aid benefit. Ear Hear. 1995 Apr; 16(2):176-86.

Di Nardo W, Cantore I, Cianfrone F, Melillo P, Scorpecci A, Paludetti G. Tinnitus modifications after cochlear implantation. Eur Arch Otorhinolaryngol. 2007 Oct;264(10):1145-9.

Han BI, Lee HW, Kim TY, Lim JS, Shin KS. Tinnitus: characteristics, causes, mechanisms, and treatments. J Clin Neurol. 2009 Mar;5(1): 11-9.

Hazell JW, McKinney CJ, Aleksy W. Mechanisms of tinnitus in profound deafness. Ann Otol Rhinol Laryngol Suppl. 1995 Sep;166:418-20.
Kim DK, Bae SC, Park KH, Jun BC, Lee DH, Yeo SW, et al. Tinnitus in patients with profound hearing loss and the effect of cochlear implantation. Eur Arch Otorhinolaryngol. 2013 May;270(6):1803-8.

Kloostra FJ, Arnold R, Hofman R, Van Dijk P. Changes in tinnitus after cochlear implantation and its relation with psychological functioning. Audiol Neurotol. 2015;20(2):81-9.

Kompis M, Pelizzone M, Dillier N, Allum J, DeMin N, Senn P. Tinnitus before and 6 months after cochlear implantation. Audiol Neurotol. 2012;17(3):161-8.

Kuk FK, Tyler RS, Russell D, Jordan H. The psychometric properties of a tinnitus handicap questionnaire. Ear Hear. 1990 Dec;11(6):434-45.

McCombe A, Baguley D, Coles R, McKenna L, McKinney C, Windle-Taylor P; British Association of Otolaryngologists, Head and Neck Surgeons. Guidelines for the grading of tinnitus severity: the results of a working group commissioned by the British Association of Otolaryngologists, Head and Neck Surgeons, 1999. Clin Otolaryngol Allied Sci. 2001 Oct; 26(5):388-93.

McCormack A, Edmondson-Jones M, Somerset S, Hall D. A systematic review of the reporting of tinnitus prevalence and severity. Hear Res. 2016 Jul;337:70-9.

Newman CW, Jacobson GP, Spitzer JB. Development of the Tinnitus Handicap Inventory. Arch Otolaryngol Head Neck Surg. 1996 Feb; 122(2):143-8.
Newman CW, Sandridge SA, Jacobson GP. Psychometric adequacy of the Tinnitus Handicap Inventory (THI) for evaluating treatment outcome. J Am Acad Audiol. 1998 Apr;9(2): 153-60.

Olze H, Szczepek AJ, Haupt H, Förster U, Zirke N, Gräbel S, et al. Cochlear implantation has a positive influence on quality of life, tinnitus, and psychological comorbidity. Laryngoscope. 2011 Oct;121(10):2220-7.

Olze H, Szczepek AJ, Haupt H, Zirke N, Graebel $S$, Mazurek B. The impact of cochlear implantation on tinnitus, stress and quality of life in postlingually deafened patients. Audiol Neurotol. 2012;17(1):2-11.

Pan T, Tyler RS, Ji H, Coelho C, Gehringer AK, Gogel SA. Changes in the tinnitus handicap questionnaire after cochlear implantation. Am J Audiol. 2009 Dec;18(2):144-51.

Quaranta N, Fernandez-Vega S, D'elia C, Filipo $\mathrm{R}$, Quaranta A. The effect of unilateral multichannel cochlear implant on bilaterally perceived tinnitus. Acta Otolaryngol. 2008 Feb; 128(2):159-63.

Quaranta N, Wagstaff S, Baguley DM. Tinnitus and cochlear implantation. Int J Audiol. 2004 May;43(5):245-51.

Ramakers GG, van Zon A, Stegeman I, Grolman $\mathrm{W}$. The effect of cochlear implantation on tinnitus in patients with bilateral hearing loss: A systematic review. Laryngoscope. 2015 Nov; 125(11):2584-92 
Rubinstein JT, Tyler RS, Johnson A, Brown CJ. Electrical suppression of tinnitus with highrate pulse trains. Otol Neurotol. 2003 May; 24(3):478-85.

Ruckenstein MJ, Hedgepeth C, Rafter KO, Montes ML, Bigelow DC. Tinnitus suppression in patients with cochlear implants. Otol Neurotol. 2001 Mar;22(2):200-4.

Spinhoven P, Ormel J, Sloekers PP, Kempen GI, Speckens AE, Van Hemert AM. A validation study of the Hospital Anxiety and Depression Scale (HADS) in different groups of Dutch subjects. Psychol Med. 1997 Mar;27(2):36370.
Stephens SD. The treatment of tinnitus-a historical perspective. J Laryngol Otol. 1984 Oct; 98(10):963-72.

Tyler RS. Patient preferences and willingness to pay for tinnitus treatments. J Am Acad Audiol. 2012 Feb;23(2):115-25.

Van de Heyning P, Vermeire K, Diebl M, Nopp P, Anderson I, De Ridder D. Incapacitating unilateral tinnitus in single-sided deafness treated by cochlear implantation. Ann Otol Rhinol Laryngol. 2008 Sep;117(9):645-52.

van Zon A, Smulders YE, Ramakers GG, Stegeman I, Smit AL, Van Zanten GA, et al. Effect of unilateral and simultaneous bilateral cochlear implantation on tinnitus: A Prospective Study. Laryngoscope. 2016 Apr;126(4): 956-61.
Vanneste S, To WT, De Ridder D. The psychometric properties of the Tinnitus Handicap Questionnaire in a Dutch-speaking population. Clin Otolaryngol. 2011 Feb;36(1):9-16.

Zeman F, Koller M, Figueiredo R, Aazevedo A, Rates M, Coelho C, et al. Tinnitus handicap inventory for evaluating treatment effects: which changes are clinically relevant? Otolaryngol Head Neck Surg. 2011 Aug;145(2):2827.

Zigmond AS, Snaith RP. The hospital anxiety and depression scale. Acta Psychiatr Scand. 1983 Jun;67(6):361-70. 